\title{
Adubação orgânica, manejo de irrigação e fertilização na produção de melão amarelo
}

\author{
Jean de O Souza; José F de Medeiros; Marcelo C de C Silva; Antônio HB de Almeida \\ UFERSA-Núcleo de Pós-Graduação, C. Postal, 137, 59625-900-Mossoró-RN; jsoliveira1@hotmail.com
}

\begin{abstract}
RESUMO
O objetivo do trabalho foi estudar o efeito da adubação orgânica e manejo de irrigação e fertilização na produção de melão amarelo. O experimento foi realizado no período de outubro a novembro de 2001, no município de Mossoró-RN. O delineamento experimental foi em blocos inteiramente casualizados, com duas repetições e os fatores foram arranjados no esquema fatorial $3 \times 4+1$. Foram estudados três tipos de materiais orgânicos (composto Ribumim ${ }^{\circledR}$, composto Integral ${ }^{\circledR}$ e esterco) em quatro doses (50; 100; 150 e 200\% do recomendado) aplicadas no plantio, e um tratamento testemunha (sem adubo orgânico), além de 3 manejos de irrigação e fertirrigação em experimentos representados pelas áreas A1, A2 e A3. Foram avaliados: produção comercial de frutos, massa media de frutos comerciais, firmeza de polpa e sólidos solúveis totais. A adição de adubo orgânico ao solo favoreceu o incremento da produção comercial de frutos nas áreas A1 e A2 e firmeza na área A1, que receberam as maiores quantidades de adubo via fertirrigação, embora essas áreas tenham produzido menos que a Área A3, onde os materiais orgânicos não influenciaram na produção.
\end{abstract}

Palavras-chave: Cucumis melo L., composto, produtividade, qualidade.

\begin{abstract}
Organic fertilization, irrigation management and chemical fertilization on yellow melon production

The effect of organic matter, irrigation handling and fertilization was evaluated on the yield of yellow melon. The experiment was carried out in Mossoró, Rio Grande do Norte State, Brazil, from October to November 2001. The experimental design was of entirely randomized blocks with two replications, in the factorial $3 \times 4 x+1$. Three organic compositions (Ribumim ${ }^{\circledR}$, integral composition ${ }^{\circledR}$ and organic manure) were studied in four levels (50; 100; 150 and 200\% of the recommended doses) applied before sowing and one control (without organic matter), besides 3 irrigation and fertilizing managements in the experiments represented by areas A1, A2 and A3. The evaluated characteristics were: commercial fruits production, average mass of the commercial fruits, pulp firmness and total soluble solids. With the addition of organic matter occurred an increment of the commercial fruits yield on the areas A1 and a2, and firmness on Area A1, in spite of the higher quantity of fertilizer through fertirrigation, even though these areas produced less than Area A3, where the organic matter had no influence on the yield.
\end{abstract}

Keywords: Cucumis melo L., composition, yield, quality.

\section{(Recebido para publicação em 12 de novembro de 2006; aceito em 21 de fevereiro de 2008)}

$\mathrm{O}$ melão (Cucumis melo L.) é uma das culturas de maior expressão econômica e social para a região Nordeste do Brasil. O estado do Rio Grande do Norte concentra a maior produção de melão, onde o pólo de desenvolvimento integrado Açú/Mossoró (Brasil, 2001) representa cerca de $63 \%$ de todo o melão produzido no estado (Silva et al., 2000). Nessa região, empregam-se altos níveis de insumos, além do uso intensivo de fertilizantes no solo (plantio e cobertura) o que pode contribuir para a degradação ambiental.

Pesquisas têm sido realizadas para se avaliar o efeito de insumos orgânicos no solo e na planta, como ferramenta importante para se definir doses, freqüência e épocas de aplicação, bem como para avaliar seus efeitos na característica final do produto (Duenhas et al., 2004).
A matéria orgânica proporciona efeitos positivos diretos no solo, como a diminuição da compactação, aumento na retenção de água, melhor estruturação do solo e maior eficiência do uso de fertilizantes, ou efeitos positivos indiretos, como o aumento geral da biomassa (MacCarthy et al., 1990; Nardi et al., 2002). Além disso, o manejo do solo com práticas que não agridam a biota e favoreçam a ciclagem dos nutrientes, tais como a utilização de esterco e compostos orgânicos, é de fundamental importância para obtenção de plantas sadias (Altieri, 1994), refletindo na produtividade e qualidade dos cultivos (Mulungoy \& Merckx, 1996).

Alguns trabalhos comprovam o efeito positivo da adubação orgânica sobre a produção e a qualidade de olerícolas como tomate, espinafre, rabanete, abobrinha italiana, cenoura, couve-flor e alface (Luz et al., 2004; Villas Boas et al., 2004), principalmente em solos de clima tropical, onde a decomposição de matéria orgânica se realiza intensamente (Silva, 2002).

Faz-se necessário testar fontes de adubo orgânico, como complemento aos métodos tradicionais de adubação, para minimizar os impactos ambientais e ecológicos do sistema de cultivo convencional, que podem causar a inviabilidade econômica da atividade (Barbiere, 2002), visando introduzir um manejo sustentável à exploração da cultura do melão na região.

O esterco tem sido uma importante fonte de matéria orgânica aplicado na cultura do melão, mas devido a problemas com plantas daninhas, nos últimos anos tem sido substituído por outras fontes alternativas, dentre elas o composto Ribumim $^{\circledR}$ e o composto Integral ${ }^{\circledR}$. O 
Ribumim $^{\circledR}$ é um produto comercial derivado de material orgânico de origem não definida (Dynia et al., 2001). O composto Integral ${ }^{\circledR}$, que corresponde ao Composto da Pole ${ }^{\circledR}$, é oriundo da mistura de casca de arroz carbonizada, pó de casca de coco e esterco bovino curtido.

Outra forma que pode causar danos ao meio ambiente no Pólo de desenvolvimento integrado Açú/Mossoró é o uso inadequado da água por meio da irrigação (fertirrigação), tanto a falta quanto o excesso de água podem afetar o desenvolvimento e a produção das culturas (Monteiro et al., 2006). Objetivouse estudar o efeito da adubação orgânica e manejo de irrigação e fertilização na produção de melão amarelo.

\section{MATERIAL E MÉTODOS}

O experimento foi conduzido na fazenda São João, de outubro a novembro de 2001, no município de Mossoró$\mathrm{RN}$, situado nas coordenadas geográficas $5^{\circ} 11^{\prime}$ de latitude sul e $37^{\circ}$ de longitude oeste, com altitude média de 18 metros. Segundo a classificação de Köppen, o clima é do tipo BSwh', apresentando temperatura média anual de $27,4^{\circ} \mathrm{C}$, precipitação pluviométrica bastante irregular, com média de 673,9 mm/ ano e umidade relativa média de 68,9\% (Carmo Filho et al., 1991).

O solo da área é classificado como Argissolo Vermelho Amarelo. Os materiais orgânicos utilizados na adubação de fundação apresentaram a composição de plantio (\% na matéria seca) de $\mathrm{N}, \mathrm{P}, \mathrm{K}, \mathrm{Ca}, \mathrm{Mg}$, Na e $\mathrm{Cl}$, respectivamente: para o material Ribumim ${ }^{\circledR}: 1,10$; 0,05; 0,20; 0,30; 0,05; 0,19 e 0,55; para composto Integral $^{\circledR}: 1,01 ; 1,00 ; 1,23$; 5,$51 ; 0,53 ; 0,32$ e 0,22 ; e para esterco: 1,37; 0,01; 0,78; 1,12; 0,42; 0,11 e 0,11. O Ribumim ${ }^{\circledR}$ apresentou 20; 560; 870; 3410 e $15 \mathrm{mg} \mathrm{kg}^{-1}$ de B, Zn, Cu, Fe e $\mathrm{Mn}$; respectivamente; o composto Integral apresentou 40; 250; 56; 8250 e 331 e o esterco 33; 69; 15; 13500 e 344.

Foram conduzidos simultaneamente três experimentos, representados pelas áreas A1, A2 e A3, realizando-se análise de variância isolada para cada área e, posteriormente, análise conjunta. O delineamento experimental utilizado em cada área foi em blocos intei- ramente casualizados, com duas repetições por área e os fatores arranjados no esquema fatorial $3 \times 4+1$, com o emprego de três tipos de materiais orgânicos (Ribumim ${ }^{\circledR}$, composto Integral ${ }^{\circledR} \mathrm{e}$ esterco) e quatro doses (50; 100; 150 e $200 \%$ do recomendado pelos produtores de melão da região de Mossoró na época, que se baseava no valor gasto com o material orgânico aplicado na adubação de fundação, estabelecido em R\$ 400,00 ha-1) além de um tratamento testemunha (sem adubo orgânico).

Cada área diferenciou-se pelas doses de $\mathrm{N}, \mathrm{P}$ e $\mathrm{K}$ aplicadas em fertirrigação e/ou pela forma em que se manejou a irrigação. Na área A1 foram adotados os procedimentos de manejo de água e fertirrigação da fazenda (110160-330 kg ha-1), em A2: 99-160-340 kg $\mathrm{ha}^{-1} \mathrm{e}$ as lâminas de irrigação foram baseadas na estimativa da ETc, segundo FAO (Allen et al., 1998), com freqüência de irrigação realizada para não deixar o potencial matricial ser inferior a -30 kPa até a floração e maturação dos frutos, e não inferior a $-15 \mathrm{kPa}$ na fase de frutificação; na área A3, o manejo da irrigação foi similar a da área A2, mas com a formulação 60-160-267. A curva de distribuição dos adubos via fertirrigação foi de acordo com a curva de absorção de nutrientes para o híbrido Yellow King (Prata, 1999).

As lâminas de águas aplicadas ao longo do ciclo, por fase fisiológica, foram: fase inicial (28,3 mm), floração (55,7 mm), frutificação (140,6 mm), e maturação (41,0 mm); inicial (29,6 $\mathrm{mm})$, floração (57,5 mm), frutificação (136,6 mm) e maturação (40,0 mm); Inicial (29,7 mm), floração (57,5 mm), frutificação (136,6 mm) e maturação (40,0 mm), para as áreas A1, A2 e A3, respectivamente.

As parcelas experimentais foram constituídas de uma fileira de plantas de $13 \mathrm{~m}$ de comprimento, utilizando-se os 6 m centrais para avaliação da produção. No plantio do melão foi utilizado o tipo “amarelo”, híbrido Goldex, da Petossed $^{\circledR}$.

O solo foi preparado por uma aração, duas gradagens, sulcamento e passagem de enxada rotativa na faixa do solo, onde foi plantado o melão. O plantio foi realizado em covas próximas aos gotejadores, com uma e duas sementes por ponto de emissão, de forma alternada, proporcionando uma densidade de 18.700 plantas por hectare. A profundidade de semeadura foi de $2 \mathrm{~cm}$. A cultura foi mantida sempre no limpo, através de capinas manuais com uso de enxada. Os frutos defeituosos foram eliminados. O controle das principais pragas e doenças foi de caráter preventivo, através de defensivos agrícolas, de acordo com os procedimentos adotados pela Fazenda São João. Os frutos foram colhidos quando se encontravam em estádio de maturação, aos 67 e aos 72 dias.

$\mathrm{Na}$ irrigação por gotejamento os emissores liberavam 1,36 $\mathrm{L} \mathrm{h}^{-1}$, trabalhando com uma pressão média de 100 $\mathrm{kPa}$, foram previamente avaliados em condições de campo, sob condições normais de operação, apresentando coeficiente de uniformidade de vazão de 85\%. Foram utilizados os coeficientes de cultivo (kc) e a evapotranspiração de referência (ETo) estimada pela equação de Penman-Monteith, proposta pela FAO, conforme sugerem Allen et al. (1998). Irrigou-se de acordo o turno de rega de cada bloco experimental, onde os volumes foram definidos mediante os dados climatológicos coletados diariamente na estação climatológica da UFERSA, aplicando-se valores em uma planilha de acordo com método da FAO (Allen et al., 1998).

Avaliou-se a produtividade comercial (tipo exportação e nacional), que correspondeu a toda a produção de frutos sem deformações. Dentro das características de qualidade, foi avaliada a firmeza da polpa (utilizando-se um penetrômetro com “pluger” de $8 \mathrm{~mm}$, quatro leituras em cada fruto, na região equatorial da polpa). O conteúdo de sólidos solúveis (obtido diretamente no suco, oriundo de fatias de melão, quatro frutos/parcela, usando método de campo com refratômetro digital ATAGO (escala de 0 a 32\%) com correção de temperatura).

\section{RESULTADOS E DISCUSSÃO}

Houve efeito significativo dos adubos orgânicos em relação à testemunha (sem uso de adubo orgânico) para a produção comercial nas áreas A1 e A2 e 
para a firmeza dos frutos na área A1 (Tabela 1). Para as doses de adubo orgânico, houve significância apenas para o Ribumim ${ }^{\circledR}$, com redução linear da produtividade com o aumento da dose $(\mathrm{Y}=$ $\left.-0,04 x+22,02 ; R^{2}=0,80\right)$ na área A2. O aumento da dose do Ribumim ${ }^{\circledR}$ pode ter provocado um desequilibro nutricional na planta, afetando assim a produção. Observou-se efeito significativo do esterco sobre a produtividade, ocorrendo incremento com o aumento da dose $\left(\mathrm{Y}=0,0402 \mathrm{x}+15,16 ; \mathrm{R}^{2}=0,97\right)$ na área A3. Esses resultados são discordantes dos obtidos por Faria et al. (1994), Faria \& Pereira (2000) e Pinto et al. (1995), em que as produtividades decresceram com aumento das doses de esterco, provavelmente devido à diferença na fonte e dose de adubo orgânico e solo.

As massas médias de frutos comerciais e sólidos solúveis totais não foram afetadas pelas fontes ou pelas doses (Tabela 1). Resultados semelhantes foram encontrados por Duenhas et al. (2004), avaliando a massa média de frutos de melão em sistema orgânico de produção, ao se testar doses, freqüência e época de aplicação do adubo. Por outro lado, Sales Júnior et al. (2003) verificaram diferença entre os materiais orgânicos em relação à testemunha, quando trabalharam com os efeitos dos mesmos adubos orgânicos sobre a qualidade do melão, embora Faria et al. (1994) não verificaram variações no teor de sólidos solúveis totais com o uso da matéria orgânica.

Para a firmeza de frutos, na área A1, houve diferença significativa entre os tratamentos com relação à testemunha (Tabela 1). Esses dados são discordantes dos obtidos por Sales Júnior et al. (2003), porém os valores encontrados neste trabalho foram superiores aos encontrados em outros trabalhos com melão (Alves et al., 2000; Soares, 2001). Observou-se efeito significativo e linear com o aumento da dose do Ribumim ${ }^{\circledR}$ sobre a firmeza dos frutos para a área A2 (Y = 0,0546 x + 30,66; $\left.\mathrm{R}^{2}=0,94\right)$.

Pela Tabela 2, verifica-se que o rendimento comercial de frutos oscilou em média de 15,00 a 19,98 t ha ${ }^{-1}$ entre as áreas e que os rendimentos na área A3 foram superiores aos obtidos nas áreas A1 e A2, que não diferiram entre si. Na

Tabela 1. Valores médios da produção comercial de frutos (PRC), massa média de frutos comerciais (MMC), firmeza de polpa (FP) e sólidos solúveis totais (SST) do melão para cada tipo de material orgânico nas três áreas. (Mean values of commercial fruits yield, mean mass of commercial fruits, pulp firmness and total soluble solids of melon fruits for each type of organic matter source in three locations). Mossoró, UFERSA, 2001.

\begin{tabular}{|c|c|c|c|c|c|}
\hline Áreas & Materiais & PRC (t ha-1) & MMC (kg) & FP (N) & SST ( ${ }^{\circ}$ Brix) \\
\hline \multirow{4}{*}{$\mathrm{A} 1$} & Testemunha & $11,94 b^{*}$ & $1,41 a$ & $37,75 b$ & $10,60 a$ \\
\hline & Ribumim $®$ & $15,83 a$ & $1,39 a$ & $41,53 a$ & $10,65 a$ \\
\hline & 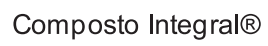 & $19,13 a$ & $1,44 a$ & $42,28 a$ & $10,52 a$ \\
\hline & Esterco & $16,67 a$ & $1,46 a$ & $40,10 a$ & $10,25 a$ \\
\hline \multirow{4}{*}{ A2 } & Testemunha & $11,43 b$ & $1,33 a$ & $37,50 a$ & $11,60 a$ \\
\hline & Ribumim $®$ & $17,02 a$ & $1,36 a$ & $37,50 a$ & $10,75 a$ \\
\hline & 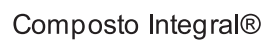 & $14,60 a$ & $1,26 a$ & $38,70 a$ & $11,95 a$ \\
\hline & Esterco & $14,45 a$ & $1,23 a$ & $39,92 a$ & $11,55 a$ \\
\hline \multirow{4}{*}{ A3 } & Testemunha & $20,15 a$ & $1,36 a$ & $35,10 a$ & $11,30 a$ \\
\hline & Ribumim ${ }^{\circledR}$ & $20,42 a$ & $1,45 a$ & $36,28 a$ & $11,80 a$ \\
\hline & 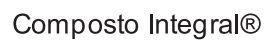 & $17,80 a$ & $1,37 a$ & $35,70 a$ & $12,45 a$ \\
\hline & Esterco & $21,50 a$ & $1,39 a$ & $35,65 a$ & $12,47 a$ \\
\hline$\overline{C V}(\%)$ & & 11,54 & 8,97 & 7,58 & 10,25 \\
\hline
\end{tabular}

*Letras diferentes nas colunas entre os materiais, para cada área, indicam diferença significativa ao nível de 5\% de probabilidade pelo teste de Tukey.

Tabela 2. Valores médios de produção comercial de frutos (PRC), massa média dos frutos comerciais (MMC), firmeza de polpa (FP) e sólidos solúveis totais (SST), nas três áreas. (Mean values of commercial fruits yield, mean mass of commercial fruits, pulp firmness and total soluble solids in three locations). Mossoró, UFERSA, 2001.

\begin{tabular}{lcccc}
\hline Áreas & PRC (t ha-1) & MMC (kg) & FP (N) & SST ('Brix) \\
\hline A1 & $17,12 b^{*}$ & $1,41 a$ & $40,91 a$ & $10,71 b$ \\
A2 & $15,00 b$ & $1,28 a$ & $36,84 a$ & $11,24 a b$ \\
A3 & $19,98 a$ & $1,39 a$ & $36,05 a$ & $11,35 a$ \\
\hline CV (\%) & 12,85 & 13,47 & 9,82 & 6,58 \\
\hline
\end{tabular}

*Letras diferentes nas colunas entre as áreas, indicam diferença significativa ao nível de $5 \%$ de probabilidade pelo teste de Tukey.

área A3 obteve-se o maior rendimento comercial com a menor dose de nitrogênio via fertirrigação (60 kg ha-1); esses resultados estão de acordo com os obtidos por Srinivas \& Prabhakar (1984), que observaram aumento de 200\% na produtividade do melão com aplicação de $50 \mathrm{~kg} \mathrm{ha}^{-1}$ desse nutriente.

Prabhakar et al. (1985), estudando a influência dos níveis de N (0; 50 e 100 $\mathrm{kg} \mathrm{ha}^{-1} \mathrm{~N}$ ), P (0 e $60 \mathrm{~kg} \mathrm{ha}^{-1}$ de $\mathrm{P}_{2} \mathrm{O}_{5}$ ) e $\mathrm{K}$ (0 e $60 \mathrm{~kg} \mathrm{ha}^{-1}$ de $\mathrm{K}_{2} \mathrm{O}$ ) na produção do melão, verificaram que houve resposta linear aos níveis de $\mathrm{N}$, com a dose de 50 kg ha-1 considerada a mais econômica. Resultados semelhantes foram verificados neste trabalho, onde a área que recebeu menor quantidade de adubo via fertirrigação (A3) foi estatisticamente superior às demais áreas, tendo em vista que não houve efeito significativo dos tratamentos dentro da área para esta va- riável. As áreas A1 e A2, que receberam as maiores doses de N-P-K via fertirrigação, obtiveram as menores produtividades de 17,12 e 15,00 t ha-1, respectivamente (Tabela 2 ). Outro fator que pode ser considerado para a área que recebeu maior adubação via fertirrigação ter apresentado menor produção comercial, foi o ataque severo da minadora (Liriomiza sp.) com maior intensidade de infestação nas áreas A1 e A2, que se encontravam mais próximas de um local com restos culturais de plantio a cerca de $30 \mathrm{~m}$, o que pode ter favorecido tal severidade. A quantidade elevada de nitrogênio aplicado nessas áreas possivelmente favoreceu a predisposição das plantas ao ataque de pragas e doenças.

Provavelmente, devido ao ciclo muito curto da cultura e ao fato de que as doses de nutrientes aplicadas em fertirrigação foram capazes de suprir 
totalmente a cultura, as doses crescentes de adubo orgânico não tenham promovido respostas positivas para a maioria das variáveis avaliadas.

Para a massa média de frutos e firmeza de polpa entre as áreas, não se observou diferença significativa. No entanto, houve significância no teor de sólidos solúveis totais ( ${ }^{\circ}$ Brix) entre as áreas. Observa-se que a amplitude oscilou de 10,71 a 11,35 (Tabela 2). De maneira geral são teores aceitos para o ponto de colheita e de comercialização (Pedrosa, 1992; Gayet, 1994). O melão com valores acima de $9^{\circ}$ Brix é comercializado no mercado externo (Sales Júnior et al., 2003).

A adição de adubo orgânico ao solo favoreceu o incremento da produção comercial de frutos nas áreas A1 e A2 e firmeza na área A1, que receberam as maiores quantidades de adubo via fertirrigação, embora essas áreas tenham produzido menos que a Área A3, onde os materiais orgânicos não influenciaram na produção (Tabela 1 ).

\section{AGRADECIMENTOS}

Agradecemos a Fazenda São João Ltda., pelo fornecimento de toda estrutura material e suporte técnico necessário e indispensável à execução do experimento.

\section{REFERÊNCIAS}

ALLEN RG; PEREIRA LS; RAES D; SMITH M. 1998. Crop evapotranspiration guidelines for computing crop water requirements. Roma: FAO (irrigation and drainage paper 56). 300p.

ALTIERI MA. 1994. Bases agroecológicas para una producción agrícola sustentable. Agricultura técnica 54(4):371-386.
ALVES RE; PIMENTEL CR; MAIA CE; CASTRO EB; VIANA FM; COSTA FV; ANDRADE GG; FILGUEIRAS HAC; ALMEIDA JHS; MENEZES JB; COSTA JG; PEREIRA LSE. 2000. Manual de melão para exportação. EMBRAPA. Brasília, DF. 51p.

BARBIERE DM. 2002. Variabilidade espacial de $\mathrm{P}, \mathrm{K}$ e soma de bases em um Latossolo vermelho eutroférrico sob cultivo de cana-deaçúcar. In: Congresso Brasileiro de Ciência do Solo. p.28.

BRASIL. 2001. Pólos de desenvolvimentos integrados. Balanço Social, 1998-2000. Fortaleza-CE. 71p.

CARMO FILHO F; ESPÍNOLA SJ; MAIA NETO JM. 1991. Dados meteorológicos de Mossoró (Janeiro de 1989 a dezembro de 1990). Mossoró: ESAM, FGD, (Coleção Mossoroense, Série C, 630). 110p.

DUENHAS LH; PINTO JM; GOMES TCA. 2004. Produtividade de melão produzido em sistema orgânico fertirrigado com substâncias húmicas extraídas de leonardita. Horticultura Brasileira 22, Suplemento. CD-ROM.

DYNIA JF; BOEIRA RC; SOUZA MD; RAIJ B. 2001. Análise de resíduos orgânicos: métodos de digestão x massas de amostras. Pesquisa em Andamento. EMBRAPA Meio AmbienteJaguariúna- São Paulo. p.1-2.

FARIA CMB; PEREIRA JR. 2000. Resposta de cultivares irrigadas à aplicação de micronutrientes no Submédio São Francisco. Pesquisa Agropecuária Brasileira 35: 12751280.

FARIA CMB; PEREIRA JR; POSSÍDIO EL. 1994. Adubação orgânica e mineral na cultura do melão em um Vertissolo do Submédio São Francisco. Pesquisa Agropecuária Brasileira 29: 191-197.

GAYET JP. 1994. Características das frutas de exportação. Ministério da Agricultura e do Abastecimento e da Reforma Agrária; secretaria de desenvolvimento rural. Brasília: EMBRAPA-SPI (Série de publicações técnicas da FRUPEX). 37p.

LUZ JMQ; BELLODI AL; MARTINS ST; DINIZ KA; LANA RMQ. 2004. Composto orgânico de lixo urbano e vermiculita como substrato para produção de mudas de alface, tomate e couve-flor. Bioscience Journal 20: 67-74.

MacCARTHY P; CLAPP CE; MALCOLM RL; BLOOM PR. 1990. Humic substances in soil and crop sciences: selected readings. Chicago: IHSS. 281p.
MONTEIRO ROC; COLARES DS; COSTA RNT; LEÃO MCS; AGUIAR JV. 2006. Função de resposta do meloeiro a diferentes lâminas de irrigação e doses de nitrogênio. Horticultura Brasileira 24: 455:459.

MULUNGOY K; MERCKX R. 1996. Soil organic matter dynamics and sustainability of tropical agriculture. Lewren: Wili-Say ce co. p.3-37.

NARDI S; PIZZEGHELLO D; MUSCOLO A; VIANELLO A. 2002. Physiological effects of humic substance on higher plants. Soil Biology \& Biochemistry 34: 1527-1536.

PEDROSA JF. 1992. Cultura do melão. Notas de aulas-Escola Superior de Agricultura de Mossoró (ESAM). Mossoró. 35p.

PINTO JM; SOARES JM; COSTA ND; BRITO LTL; PEREIRA JR. 1995. Aplicação de N e K via água de irrigação em melão. Horticultura Brasileira 13: 192-195.

PRABHAKAR BS; SRINIVAS K; SHUKLA V. 1985. Yield and quality of muskmelon. (cv. Haramadhu) in relation to spacing and fertilization. Progressive Horticulturae 17: 51-55.

PRATA EB. 1999. Acumulação de biomassa e absorção de nutrientes por híbridos de meloeiro (Cucumis melo L.). Fortaleza: UFC. 59p (Tese mestrado).

SALES JÚNIOR R; AMARO FILHO J; MOTA JCA; NUNES GHS; PEREIRA EWL. 2003. Efeito do Ribumim ${ }^{\circledR}$ nas características póscolheita do melão amarelo “AF 646”. Horticultura Brasileira 21: 98-100. Suplemento CD-ROM.

SILVA GG; PRAÇA EF; MENEZES JB; GOMES JUNIOR J; VIEIRA CPG. 2000. Qualidade de híbridos de melão após a aplicação de imidacloprid para controle de mosca-branca. Horticultura Brasileira 18: 179-182.

SILVA MA. 2002. Influência de nitrogênio e matéria orgânica na produção de melão (Cucumis melo L.). Campina Grande: UFPB. 53p. (Tese mestrado).

SOARES SPF. 2001. Qualidade do melão (Cucumis melo L.) exportado pelo porto de Natal-RN. Mossoró: ESAM. 55p. (Monografia graduação).

SRINIVAS K; PRABHAKAR BS. 1984. Response of muskmelon (Cucumis melo L.) to varying levels of spacing and fertilizers. Singapore Journal of Primary Industries 12: 56-61.

VILLAS BOAS RL; PASSOS JC; FERNANDES DM; BULL LT; CEZAR VRS; GOTO R. 2004. Efeitos de doses e tipos de compostos orgânicos na produção de alface em dois solos sob ambiente protegido. Horticultura Brasileira 22: 28-34. 